\title{
Multilevel Linear Fm Pulse Compression Radar Signals Simulator
}

\author{
Mahmod A. Al-Zubaidy, PhD \\ Mosul University, \\ Mosul, Iraq
}

\author{
Samaa K. Al-Saffar \\ Mosul University, \\ Mosul,Iraq
}

\begin{abstract}
The radar signals simulators became an essential requirement for the development and the evaluation of the performance of the radar systems. Many types of the radar signals simulators were implemented using different techniques. Some of them use digital electronics boards and digital electronics cards as help access to real-world signals and instrumentation for test different types of radar systems, but these types of simulators did not has the ability to generate the radar signals in the intermediate frequency (IF) stage. In this paper, the radar signals simulator in video stage and (IF) stage using PC, arbitrary waveform generator card (DA4300) and National Instruments Digital Electronic Field programmable Gate Array (NI-FPGA) board was proposed. In addition of the hardware requirements, LabVEIW program was used with the FPGA board to generate some of the radar signals such as the synchronization signal (SYNC), Antenna Location signals (ACP1, ACP2 (Angle Clock pulse) and NP (North Pulse)), and others, while Microsoft Visual C++ software was used with the (DA4300) card to generate a transmitted signal, target signal, and other signals in (IF) and video stages. The proposed simulator system has the ability to generate the signals for different types of radars; one of these types is the pulse compression radar. The generation of linear FM pulse compression radar signals is compared with the MATLAB Simulink results for this type of radar.
\end{abstract}

\section{Keywords}

Linear FM Pulse Compression Radar, MATLAB Simulink, Radar Signal Simulator, FPGA, DA4300 Card, Pulse Compression Filter \& LABVIEW.

\section{INTRODUCTION}

In the radar systems, one of the most important parameters which affect the target detection probability is the average of power for the transmitted signal as shown in equation (1).

$$
\mathrm{P}_{\mathrm{e}}=\frac{\mathrm{P}_{\mathrm{Av}} \cdot \mathrm{G}^{2} \cdot \sigma \cdot \lambda^{2}}{(4 \cdot \pi)^{3} \cdot \mathrm{R}^{4}}
$$

where

$\mathrm{P}_{\mathrm{e}}=$ received power.

$\mathrm{P}_{\mathrm{Av}}=$ average transmitted power.

$\sigma=$ target cross section $\left[\mathrm{m}^{\wedge} 2\right]$.

$\mathrm{G}=$ antenna gain.

$\mathrm{R}=$ target range $[\mathrm{m}]$.

$\lambda=\mathrm{RF}$ signal wavelength $[\mathrm{m}]$.

In the pulse radars, the average transmitted power will change depending on the pulse duration $(\tau)$, pulse repetition frequency
(PRF), and the peak transmitted power, the relationship of the average transmitted power with the pulse duration and peak power transmitted is shown in the following equation [1]:

$$
P_{A v}=\frac{P_{t} \cdot T_{p}}{T}
$$

where

$\mathrm{P}_{\mathrm{t}}$ : - the peak transmitted power.

$\mathrm{T}_{\mathrm{p}}$ : - pulse duration.

$\mathrm{T}$ : - period of pulse where $\mathrm{T}=1 /(\mathrm{PRF})$.

The range resolution is defined as the ability of a radar system to distinguish between two or more targets on the same bearing angle but at different ranges. The degree of the range resolution depends on the width of the transmitted pulse. The theoretical range resolution of a pulse radar system can be calculated from the following equation [2]-[3]:

$$
\Delta R=\frac{T_{p} \cdot c}{2}
$$

Where $\Delta \mathrm{R}$ : - range resolution. $\mathrm{c}:-$ the velocity of the light.

The pulse compression radar is one of the basic types of radars used for improving the detection probability of the targets and increased the range resolution of the radar as well. This radar use the technique of transmission of a modulated or coded long duration pulses and with apply some processing to improve the received (echo) signal on the receiver part. A high range resolution due to narrow duration pulses can be obtained [4]. This modulation or coding transmitted pulses can be either:

1- FM (frequency modulation) which includes:

- Linear (chirp radar).

- Non-linear.

- Time-frequency-coded waveform (e.g. Costas code).

2 - PM (phase modulation).

2.LINEAR FREQUENCY MODULATION (LFM) PULSE COMPRESSION RADAR

The block diagram of linear FM pulse compression radar is shown in figure (1). 


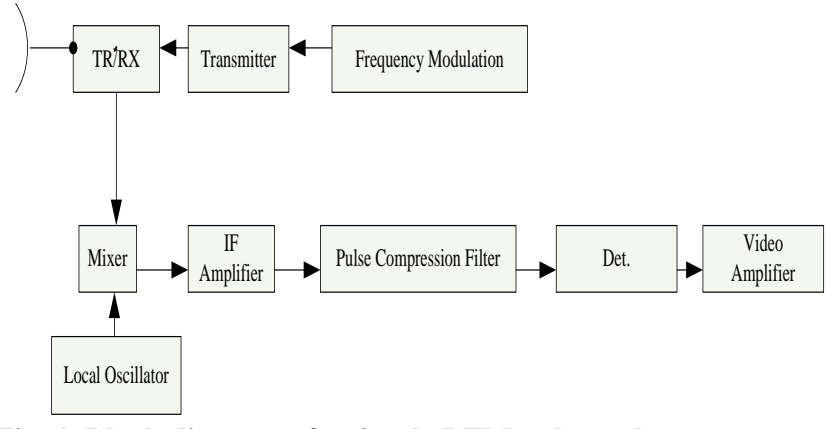

Fig. 1 Block diagram of a simple LFM pulse radar system

In the pulse compression method, the pulses will be transmitting after modulated with a linear FM signal as shown in figure (2). This method has an advantage that the hardware still relatively simple [1] and the resolution due to narrow duration pulses can be obtained [4].

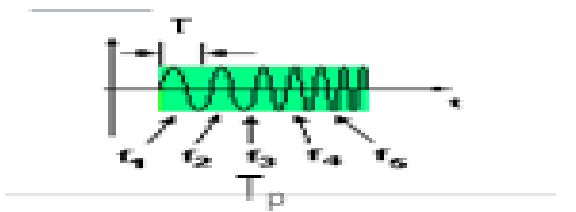

(A)

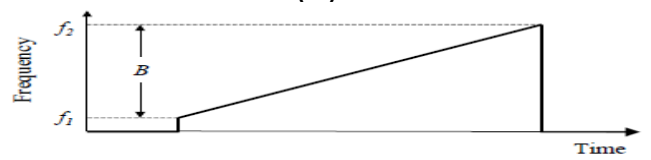

(B)

Fig. 2 (A) Pulse compression signal (uncompressed pulse) in time domain.(B) The frequency of the same pulse over time.

The pulse compression ratio can be defined as the ratio of the long pulse width $T_{p}$ to the compressed pulse width $\tau,\left(T_{p} / \tau\right)$. The bandwidth $\mathrm{B}$ and the compressed pulse width $\tau$ are related as $\mathrm{B}=1 / \tau$. The pulse compression ratio can be written [5].

Pulse compression ratio $=\mathrm{B} * \mathrm{~T}_{\mathrm{p}}$

where

B: the bandwidth of the modulation within each pulse in hertz. $\mathrm{T}_{\mathrm{p}}$ : the uncompressed pulse width in seconds.

The pulse comprission Filters that used in the linear FM pulse compression radars can be implemented by two main way;

a) Digital processing with (A/D) conversion.

b) surface acoustic wave filter (SAW).

The operation principles of a pulse compression filter in (SAW) are illustrated in figure (3). The pulse compression filter contain the delay lines units, bank of band pass filters units and summation unit. The compression filter allows the end of the pulse to catch up to the beginning and produces a narrower output pulse with higher amplitude. The output of the compression filter signal consists of the compressed pulse accompanied by responses at other times sidelobes and the width of the compressed pulse $=1 / \mathrm{B}=\tau$ and delay with pulse duration Tp. Figure (4) shows a view of the compressed pulse of a linear FM radar in time domain [6].

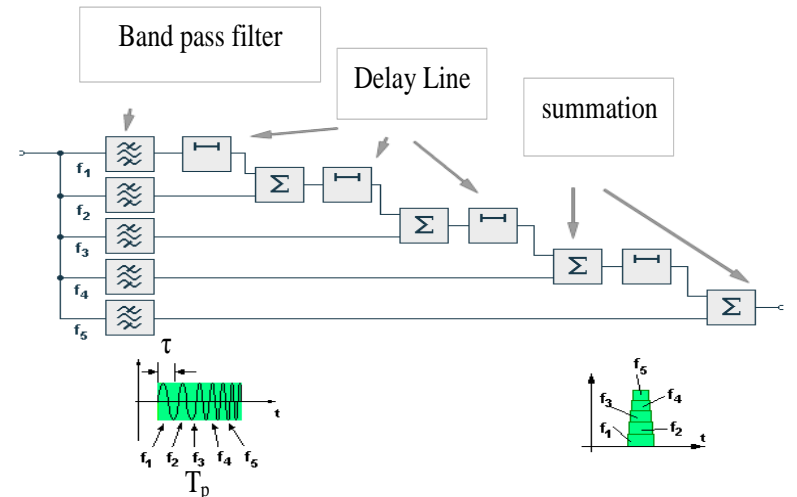

Fig. 3 The principle of pulse compression filter

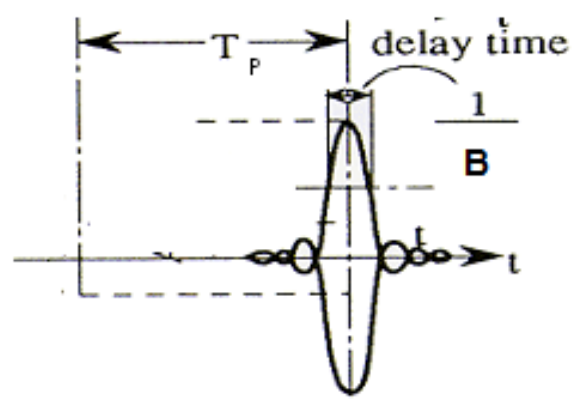

Fig. 4 The output of pulse compression filter

\section{MATLAB SIMULATION SYSTEM FOR THE LINEAR FM PULSECOMPRESSION RADAR}

The linear FM pulse compression radar was simulated by using MATLAB Simulink to generate the required signals. The main blocks for the linear FM pulse compression radar was created from basic blocks in MATLAB Simulink and modified in to one block such as antenna location signals block, target block and pulse compression filter block. Figure(5) shows the MATLAB Simulink for this radar.pulse $=1 / B=\tau$ and delay with pulse duration Tp. Figure (4) shows a view of the compressed pulse of a linear FM radar in time domain [6].

\section{HARDWARE IMPLEMENTATION OF THE LINEAR FM PULSE COMPRESSION RADAR SIGNALS SIMULATOR}

The radar signals simulator for pulse compression radar has been implemented through two hardware units in addition to the PC and RF switch. The first unit is the FPGA board where the software was built with LabVIEW VIs language to generate some signals such as synchronization signal, ACP1, ACP2, north pulse (NP), and the gate signal. The duration of the NP signal is one ACP clock signal; this pulse appears when the radar antenna is in the real geographic north, while ACP1 and ACP2 each one has 4096 square pulses per radar antenna turn gives indication about the angle of the target. The gate signal represented as a pulse which appears the target in the specific direction; therefore, it has the duration equal to the duration of the detection of targets. The second unit is the arbitrary waveform generator card (DA4300) where the software was built with Microsoft Visual C++ (MSVC) language to generate the transmitted signal and target signal at IF and video stages for different cases. The block diagram of this system and the photograph of the implemented hardware system are shown in figures (6) and (7). 


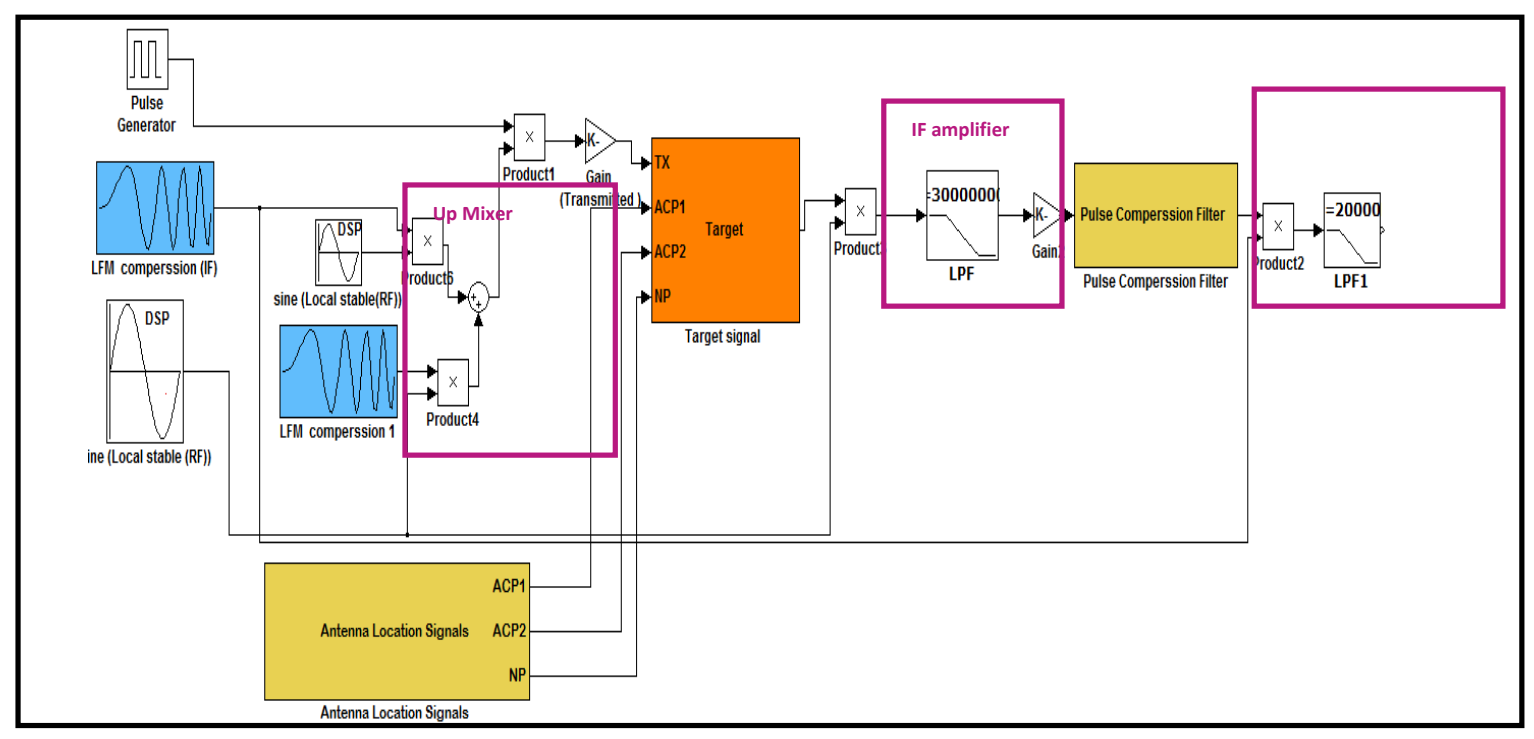

Fig. 5 The linear FM pulse compression radar in MATLAB Simulink

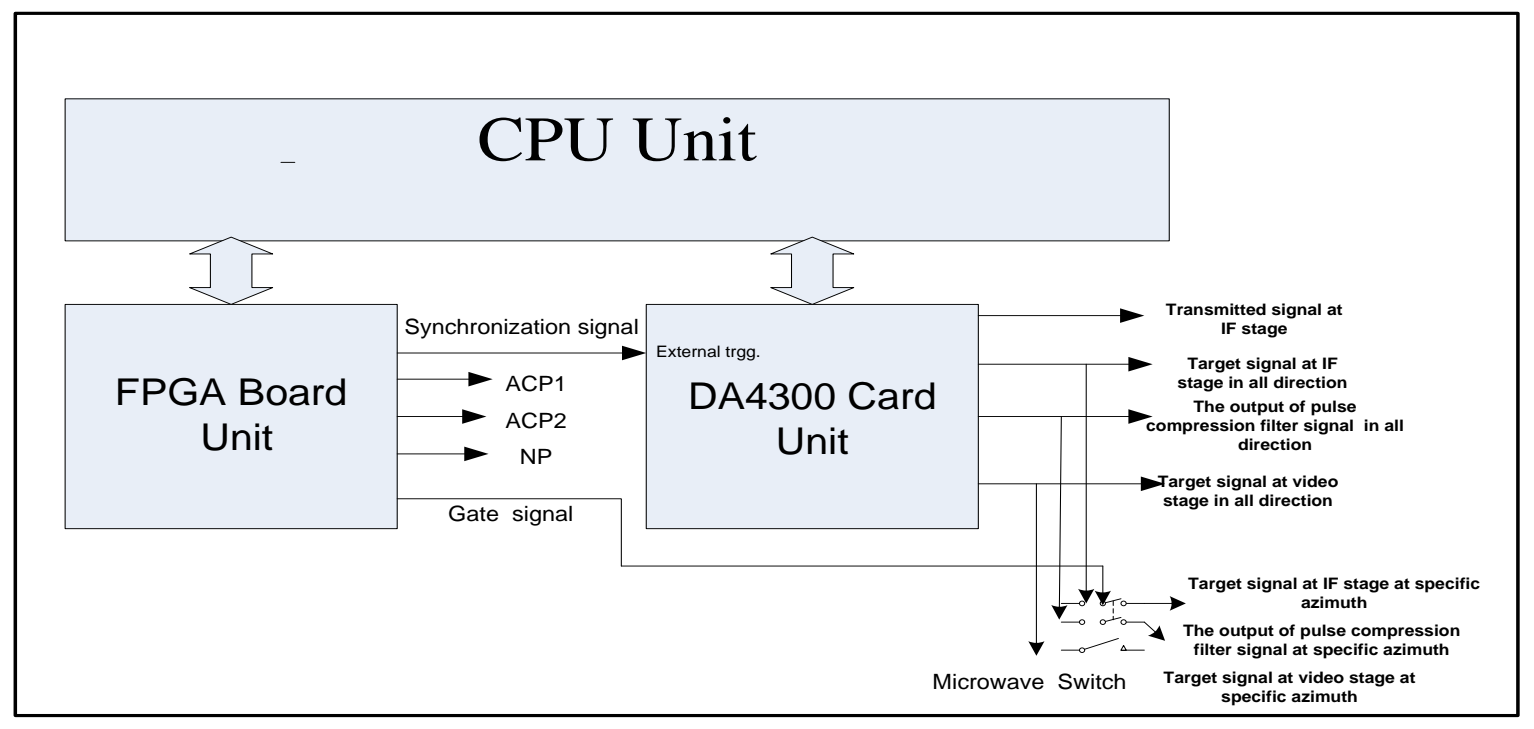

Fig. 6 Block Diagram of the hardware implementation for the linear FM pulse compression radar signals simulator

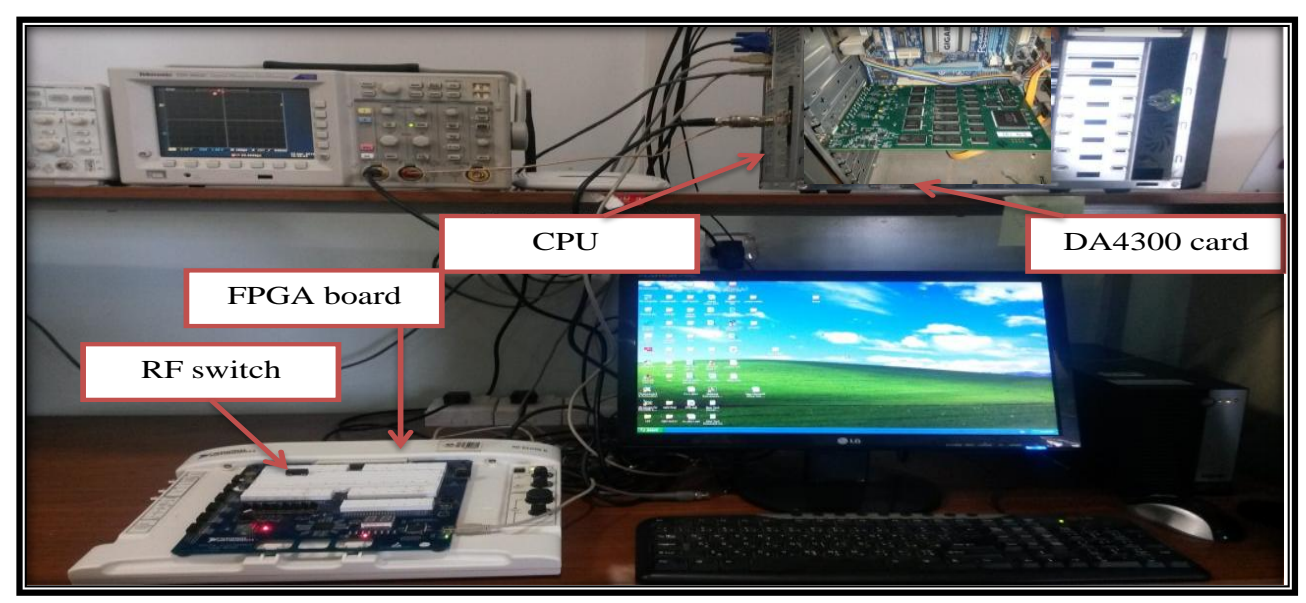

Fig. 7 The practical implementation of hardware for the linear FM pulse compression radar signals simulator 


\subsection{FPGA Board Unit}

The NI Digital Electronics FPGA Board is a circuit development platform based on the XC3S500E Xilinx Spartan-3E FPGA. The board includes the necessary I/O for designing the advanced digital electronic circuits [7]

In the presented work of this unit, the required signals generation system had been designed using the basic blocks in the LABVEIW Vis FPGA as shown in figure (8) and then the design system are downloaded onto the GPIO lines to the FPGA chips. The front panel of the LABVEIW VIs for this design that represented the inputs parameters for the period pulse,such as pulse width, number of turns per minute and the start and stop of azimuth parameters as shown in figure (9).

\subsection{The Arbitrary Waveform Generator Card (DA4300)}

The DA4300 is the fastest PCI based arbitrary waveform generator that has a (4) channel, 12-bit, $300 \mathrm{M}$ sample/second arbitrary waveform generator can be working with internal or external trigger on a single mid-sized PCI card [8].

The card had been programmed by Microsoft Visual C++ (MSVC) language depending on the specifications of pulse compression radar. Four channels had been exploited to generate the transmitted signal, pulse compression filter signal and target signal at IF and video stages for fixed target and moving target. The SYNC signal that generated in by the FPGA board entered as an external trigger to the DA4300card in order to generate the transmitted signal and target signal at specific time (specific range).

In fact the generated target signals will appear in all directions from the Da4300 card. Therefore, the gate signal had been generated by the FPGA board controlling microwave switch to show the target signals in the certain direction and certain duration only.

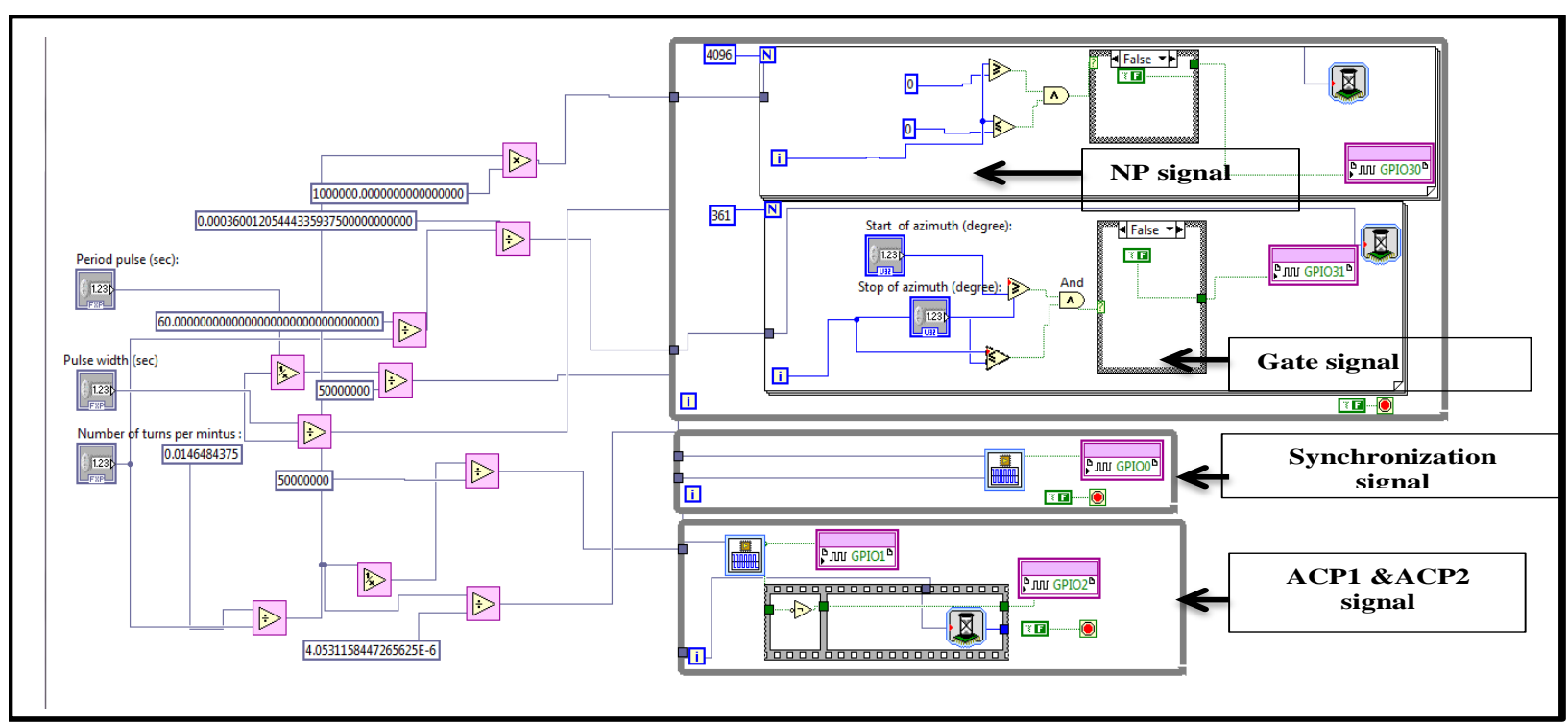

Fig. 8 Block diagram of the designed in LABVIEW of FPGA board

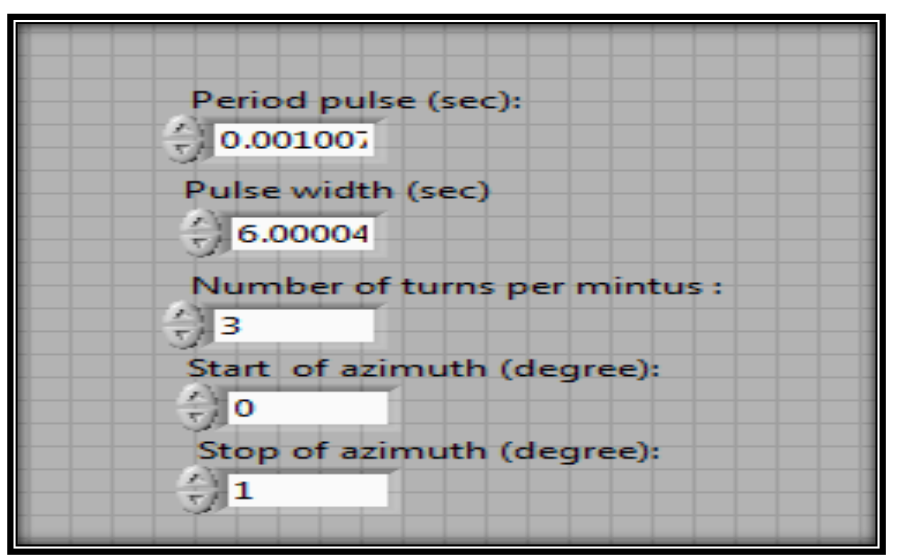

Fig. 9 The front panel of the designed in LABVIEW of FPGA board 


\section{SIMULATION RESULTS FOR MATLAB}

The pulse compression radar was simulated in the MATLAB Simulink under the following parameters that stated in table 1.

Table 1. The input parameters of pulse compression radar

\begin{tabular}{|c|c|}
\hline \multicolumn{2}{|c|}{ Transmitted Signal } \\
\hline Period of pulse & $1 \mathrm{msec}$ \\
\hline Pulse width & $100 \mu \mathrm{sec}$ \\
\hline IF frequency & $30 \mathrm{MHz}$ \\
\hline Consider RF frequency & $170 \mathrm{MHz}$ \\
\hline Deviation frequency & $10 \mathrm{KHz}$ \\
\hline Bandwidth & $200 \mathrm{KHz}$ \\
\hline Range resolution & $750 \mathrm{~m}$ \\
\hline Target signal \\
\hline The velocity of moving target & $500 \mathrm{~m} / \mathrm{sec}$ \\
\hline The velocity of Fixed target & 0 \\
\hline Movement target angle & $60 \mathrm{degree}$. \\
\hline Wavelength & $1.5 \mathrm{~m}$. \\
\hline Range for fixed target & $40 \mathrm{Km}$ \\
\hline Range for moving target & $100 \mathrm{Km}$ \\
\hline Azimuth & $(0-0.08) \mathrm{degree}$. \\
\hline
\end{tabular}

Figure (10-A) shows the MATLAB Simulink results, one can be seen that the transmitted signal will be returned with two echoes, one of echoes is for fixed target and the other for moving target. also It can be observed that the fixed target signals at IF and video stages affected by a delay and phase shift. This phase shift is the same at each received pulses. While the moving target signal will be affected by the delay, Doppler shift and different phases at each received pulse. The output of the pulse compression filter signal of moving and fixed targets appears as a compressed pulse with side lobes. The width of the compressed pulse is equal $1 / \mathrm{B}(5 \mu \mathrm{s})$ which is smaller than the pulse width of the transmitted signal, the peak of compressed pulse is delayed by Tp $(100 \mu \mathrm{s})$. There is only one difference of pulse compression filter signal between the fixed and moving target is the carrier frequency.

The video signal appears as a sinc function. For a fixed target,there is clear that this video signal has the same amplitude and phase, while in moving target, it appears with different amplitude and phase, in this cases the target signal of different stages appearing within all 360 degree (all directions). Figure (10-B) shows the antenna location signals that represent ACP1, ACP2 and NP. While figure (10-C) shows the target signal of different stages at specific azimuth (0-0.08) degree.

\section{SIMULATION RESULTS FOR HARDWARE IMPLEMENTATION}

The implemented hardware of the pulse compression radar simulator was tested under the parameters that shown in table (2) and table (3).
Table 2. The input parameters of the generated signal from FPGA Board

\begin{tabular}{|c|c|}
\hline \multicolumn{2}{|c|}{ Synchronization Signal } \\
\hline Period of pulse & $1 \mathrm{msec}$ \\
\hline Pulse width & $100 \mu \mathrm{sec}$ \\
\hline Antenna location signal \\
\hline Number of turns per minute & 3 turns \\
\hline Movement direction of antenna & Clockwise \\
\hline \multicolumn{2}{|c|}{ Gate Signal } \\
\hline Azimuth of the target & $(10-20)$ degree \\
\hline
\end{tabular}

Table 3. The input parameters of the generated signal from DA4300 Card

\begin{tabular}{|c|c|}
\hline Transmitted Signal \\
\hline Period of pulse & $1 \mathrm{msec}$ \\
\hline Pulse width & $100 \mu \mathrm{sec}$ \\
\hline Consider RF frequency & $170 \mathrm{MHz}$ \\
\hline IF frequency & $30 \mathrm{MHz}$ \\
\hline Deviation frequency & $10 \mathrm{KHz}$ \\
\hline Bandwidth & $200 \mathrm{KHz}$ \\
\hline Range resolution & $750 \mathrm{~m}$ \\
\hline Target signal \\
\hline Doppler frequency of moving target & $333.33 \mathrm{~Hz}$ \\
\hline Doppler frequency of Fixed target & 0 \\
\hline Range for fixed target & $40 \mathrm{Km}$ \\
\hline Range for moving target & $100 \mathrm{Km}$ \\
\hline
\end{tabular}

Figure (11) shows the SYNC, ACP1, ACP2, NP and Gate signals respectively. These signals are generated by the FPGA board depending on the parameters stated in the table (2).

Figure (12) and figure (13) show the transmitted signal and the fixed and moving targets signal at IF and video stages which generated using the (DA4300) card depending on the stated parameters in table (3). It can be observed clearly from figure (12) that the fixed target signal will be affected by a delay and phase shift at IF stage, while the moving target signal will be affected by a delay, Doppler shift and the phase shift which was changing at each pulse received. The output of pulse compression filter signals has been generated from DA4300 card for moving and fixed targets are similar to MATLAB Simulink result. It can be observed that the hardware implementation results signal appear as a compressed pulse with sidelobes. The width of the compressed pulse is equal $1 / \mathrm{B}(5 \mu \mathrm{s})$. The peak of compressed pulse is delayed by $\mathrm{Tp}(100 \mu \mathrm{s})$ as shown in figure (13). Also, it can be observed clearly from figure (14) the video signals, it appear as a sinc function with the same amplitude and phase for fixed target at each pulse received, while in the moving target, the video signal appears with different amplitude and phase at each received pulse that will be called a butterfly. Figure (15) shows that the target signal which appears in the specific azimuth (10-20) degree depending on the gate signal that is synchronized with the NP signal. 


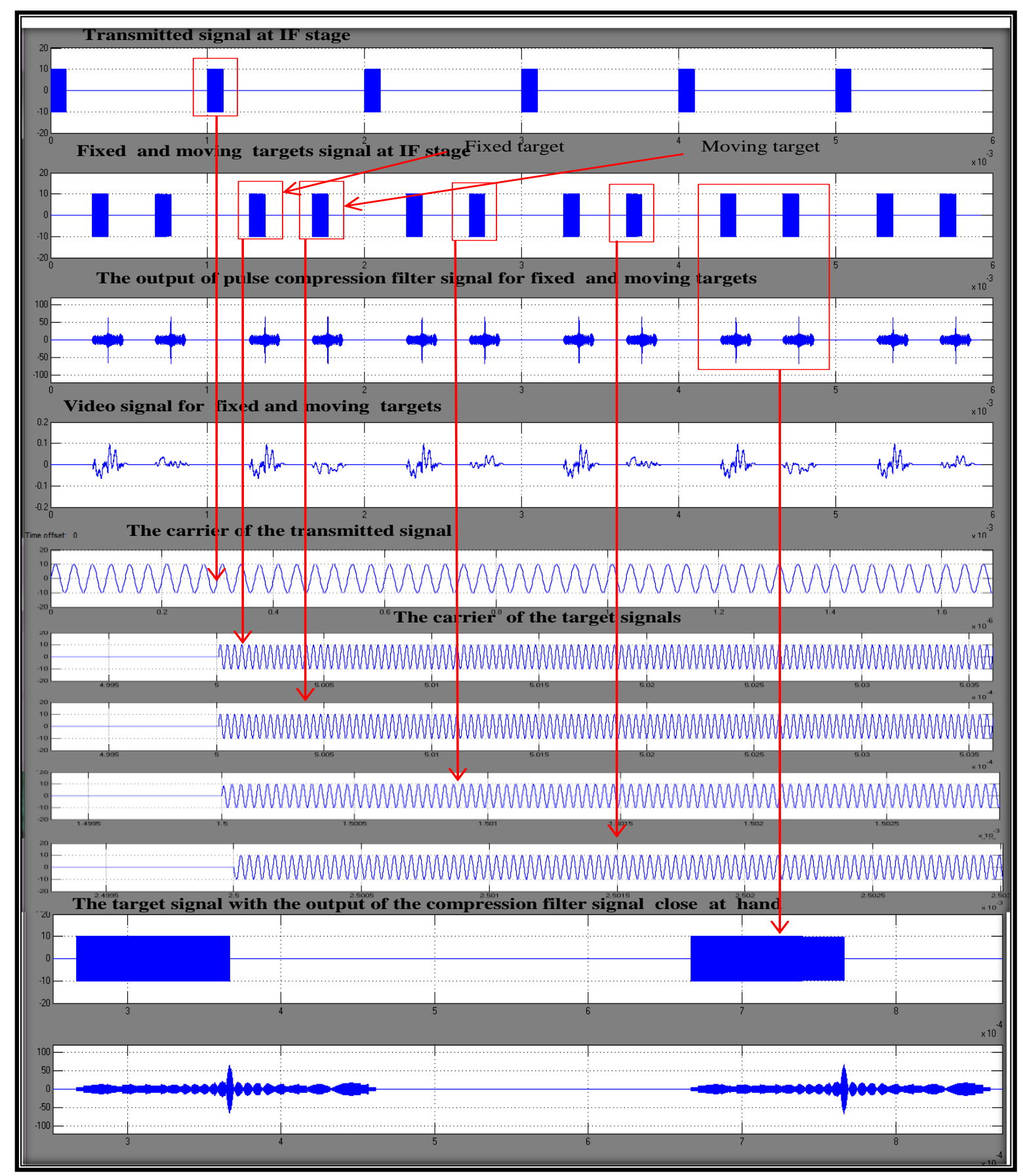

Fig. (10 - A) The MATLAB Simulink results for the Transmitted signal and target signal at IF and video stages and pulse compression filter signal for the fixed and moving targets.

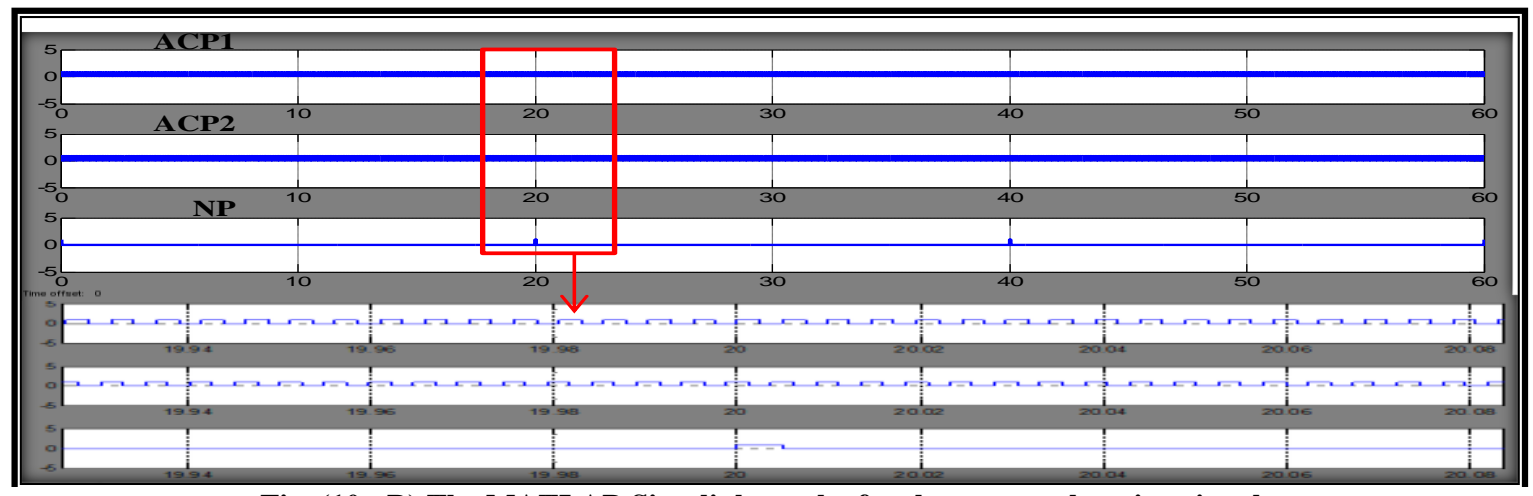

Fig. (10 - B) The MATLAB Simulink results for the antenna location signals 


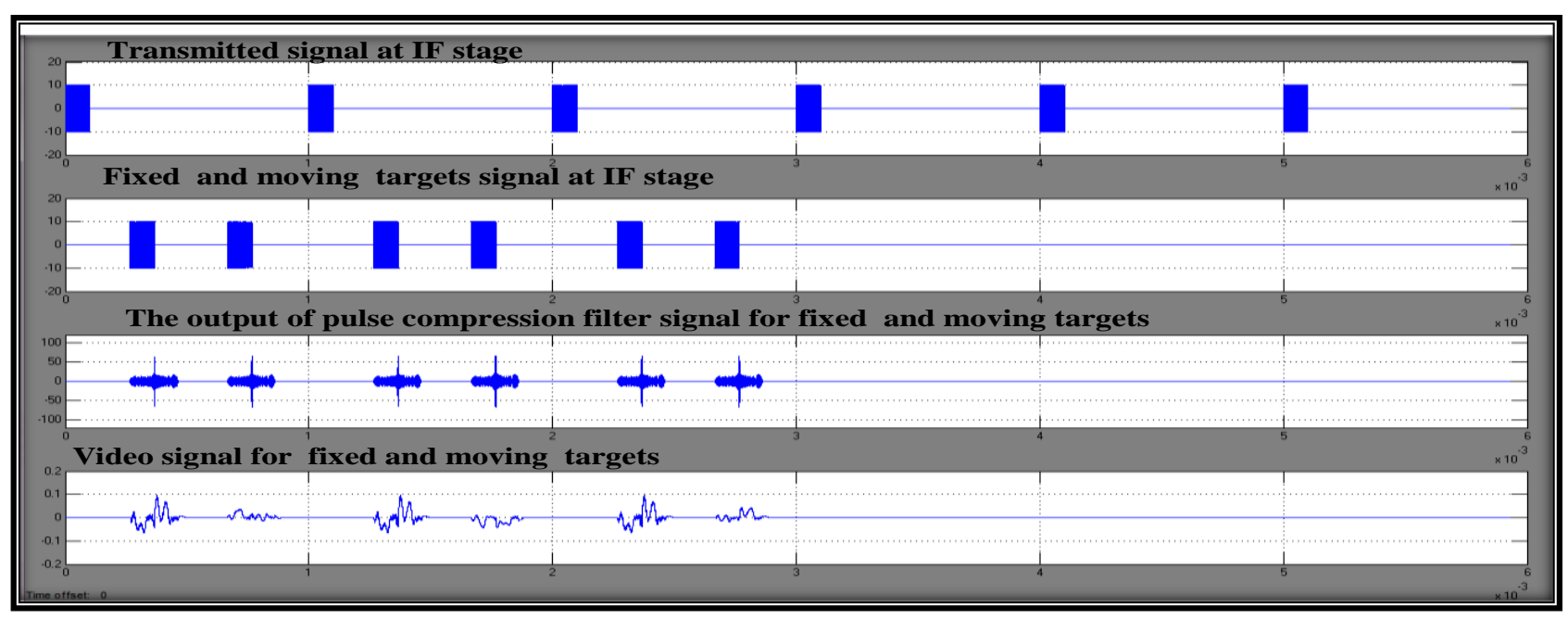

Fig. $(10$ - C) The MATLAB Simulink results for the Transmitted signal and target signal at IF and video stages and pulse compression filter signal for the fixed and moving targets at specific azimuth

Fig. 10 The MATLAB Simulink results.

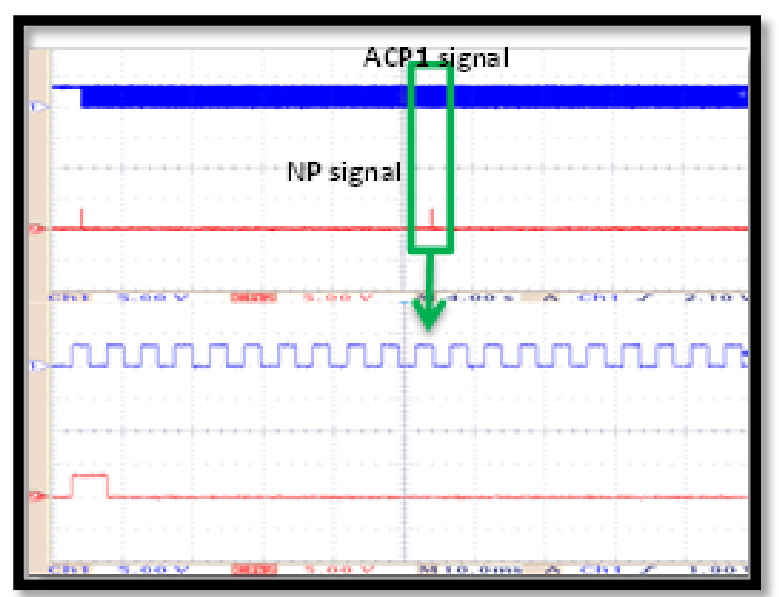

Fig. (11-A) Synchronization signal

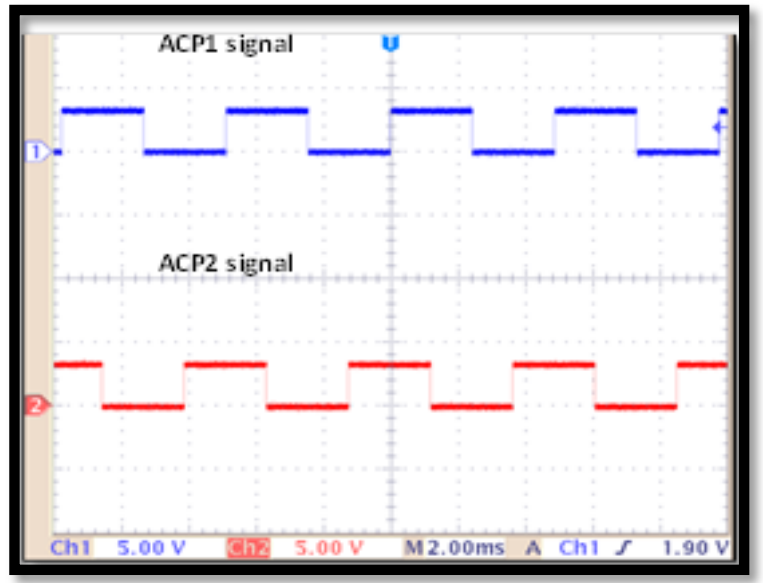

Fig. (11-B) ACP1\& ACP2 signals

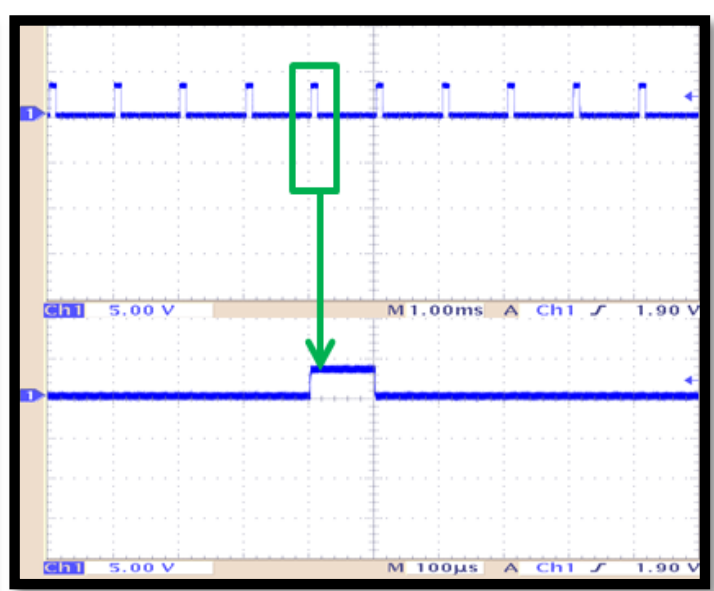

Fig.(11-C) ACP1 \& NP Signals

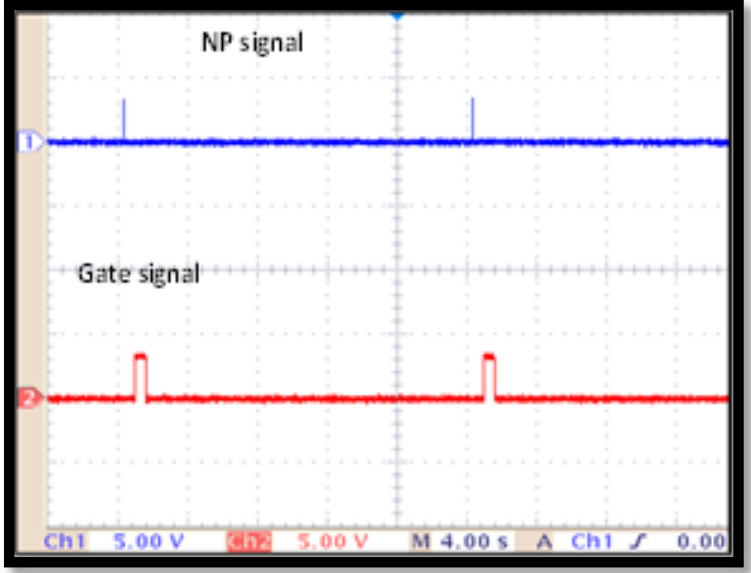

Fig. (11-D) NP and Gate signals

Fig. 11: Hardware simulation Generated signals on FPGA board 


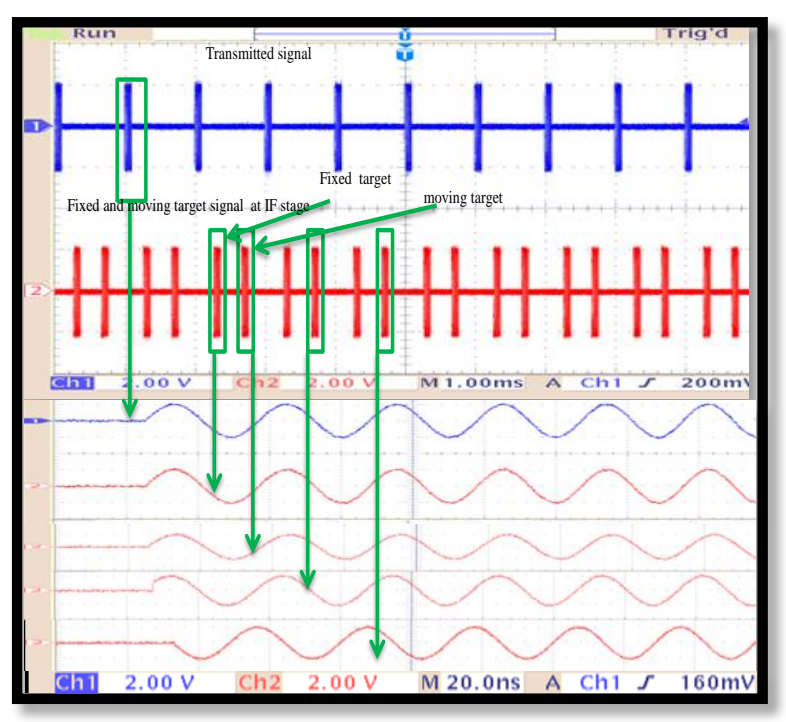

Fig. 12Transmitted signal and the fixed and moving targets signals

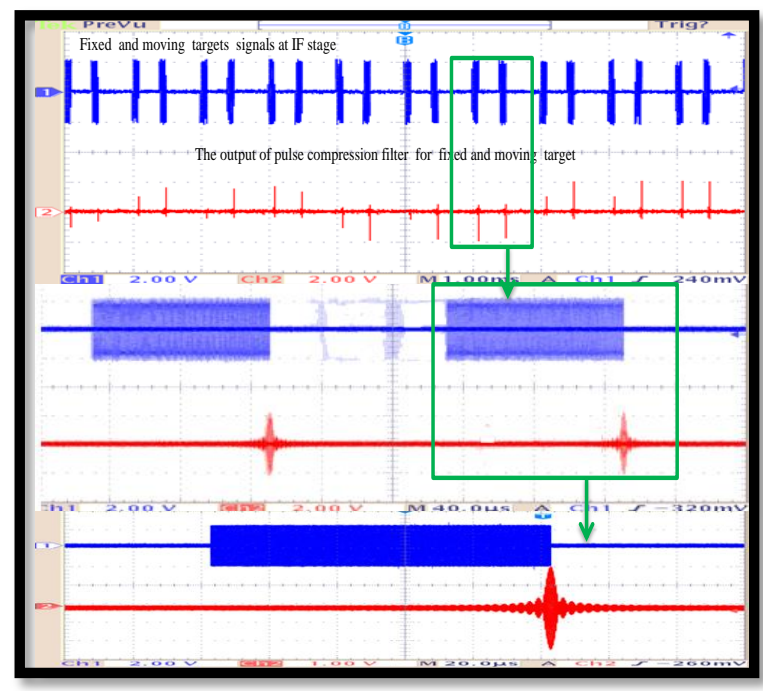

Fig. 13 Target signals at IF stage and the output of pulse compression filter for the fixed and moving targets

\section{CONCLUSIONS}

The pulse compression radar signals simulator was successfully designed and implemented using FPGA and DA4300 Card with LABVIEW VIs FPGA and Microsoft virtual C++ (MSVC) languages in hardware and it also was successfully simulated using MATLAB Simulink. In the hardware implementation, the radar system simulator has the flexibility to generate more than one target with different ranges, different direction, different speed and direction of movement. It can be used successfully for examining or developing different types of signal processing for pulse compression radar and other types of radars.

\section{REFERENCES}

[1] M. I. Skolnik, "Introduction to radar systems," 2nd Edition, McGraw-Hill, 1981.

[2] D. K. Barton, and S. A. Leonov, "Radar Technology Encyclopedia", Artech House, 1998.

[3] US Navy, Aviation Electricity And Electronics, NAVEDTRA 14339.

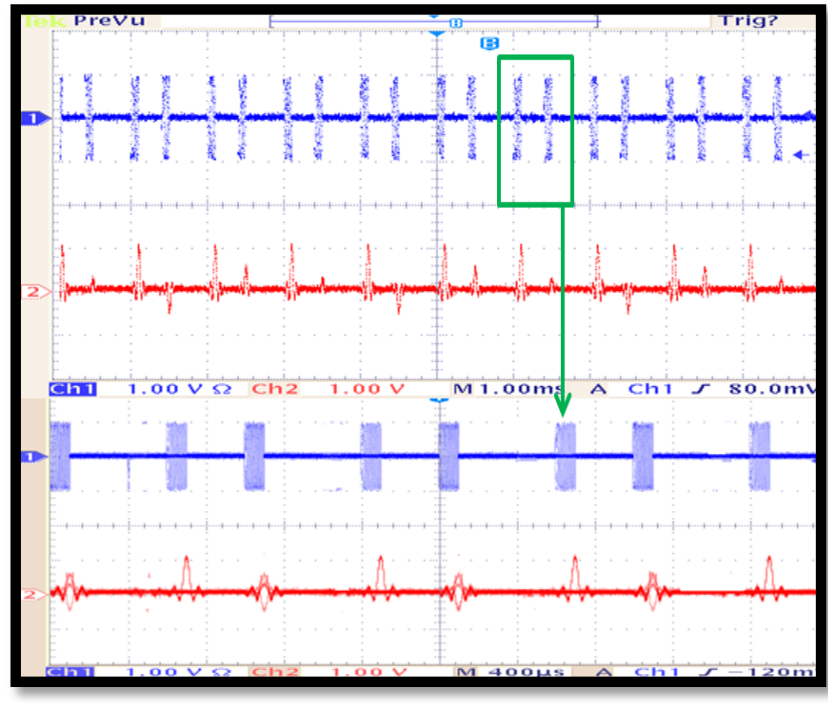

Fig. 14 Target signals at IF and video stages for the fixed and moving targets

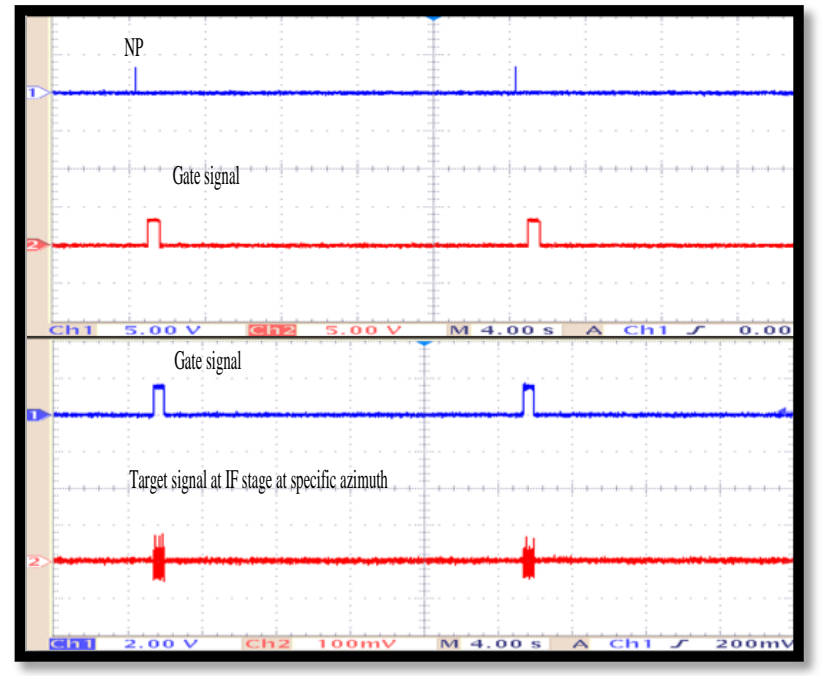

Fig. 15 Gate signal and target signal at IF and video stages for the fixed and moving targets at specific azimuth

[4] M. I. Skolnik, "Radar Handbook," 2nd Edition, McGrawHill, 1990

[5] M. I. Skolnik, "Introduction to radar systems," 3rd Edition, McGraw-Hill, 2001.

[6] Christian Wolff, Text is available under the GNU Free Documentation License, and theCreative Commons Attribution Share Alike 3.0 Unported license. http://www.radartutorial.eu/08.transmitters/Intrapulse $\% 20 \mathrm{M}$ odulation.en.html

[7] Ursutiu, Doru. "FPGA LabVIEW Programming, Monitoring and Remote Control", InternationalJournal of Online Engineering/18612121, 20090501.

[8] Data sheet,"DA4300 300 MSPS, 4-Ch, 12-bit, PCI Arbitrary Waveform Generator, " Chase Scientific Company. 